
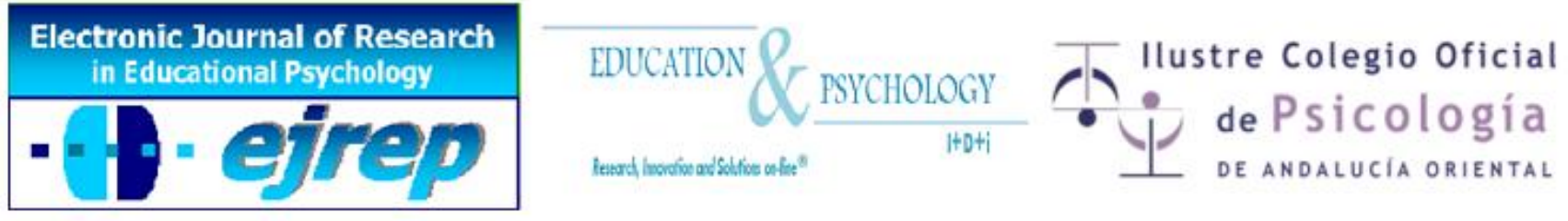

\title{
Incidencia del Funcionamiento Ejecutivo en el Rendimiento en Comprensión Lectora en Adolescentes
}

\section{María Silvina Demagistri ${ }^{1}$, María Marta Richards ${ }^{1,2}$ $\mathbf{y}$ Lorena Canet Juric $^{1,2}$.}

${ }^{1}$ Centro de Investigación en Metodología, Memoria, Educación y Procesos Básicos, Facultad de Psicología, Universidad Nacional de Mar del Plata

${ }^{2}$ Consejo Nacional de Investigaciones Científicas y Técnicas (CONICET), Mar del Plata

\section{Argentina}

Correspondencia: María Silvina Demagistri. Funes 1477. CP 7600. Mar del Plata, Argentina. E-mail: msdemagistri@gmail.com

(C) Education \& Psychology I+D+i and Ilustre Colegio Oficial de Psicología de Andalucía Oriental (Spain) 


\section{Resumen}

Introducción. La comprensión lectora es una habilidad cognitiva compleja que se ha asociado a procesos ejecutivos como la memoria de trabajo (MT) y la inhibición. Dado que el desarrollo de estas habilidades continúa hasta la adolescencia tardía, esta investigación propone estudiar el rol que sobre los distintos niveles de rendimiento en comprensión lectora ejercen ambos procesos en adolescentes de dos franjas etarias.

Método. Se evaluó el desempeño en comprensión de textos, MT, inhibición, fluidez lectora y habilidades verbales en 104 adolescentes (12/13 años $n=53 ; 17 / 18$ años $n=51)$.

Resultados. Se encontró que los desempeños en comprensión lectora, MT e inhibición presentan incrementos significativos según la edad. A su vez, la correlación parcial mostró que la comprensión lectora se asocia a: habilidades verbales, MT e inhibición. Por último, las pruebas de diferencia de grupos señalan que los estudiantes con alto y bajo rendimiento en comprensión difieren significativamente en habilidades verbales y funcionamiento ejecutivo en la franja 12/13 años. En cambio, sólo difieren significativamente en habilidades verbales en la franja 17/18 años.

Conclusión. La comprensión lectora, la MT y la inhibición mejoran durante la adolescencia. Sin embargo, la relación entre estas habilidades difiere según el momento etario considerado.

Palabras Clave: Memoria de trabajo, procesos inhibitorios, comprensión lectora, adolescentes. 


\title{
Incidence of Executive Processes on Reading Comprehen- sion Performance in Adolescents.
}

\begin{abstract}
Introduction. Reading comprehension is a complex cognitive skill that has been associated with executive functions such as working memory (WM) and inhibition. Given that the development of these abilities continues through late adolescence, this study seeks to explore the role that both processes play with respect to varying levels of reading comprehension performance in adolescents in two age groups.
\end{abstract}

Method. We evaluated performance in the comprehension of texts, WM, inhibition, reading fluency and verbal skills in 104 adolescents $(12 / 13$ years of age $n=53 ; 17 / 18$ years of age $n=$ $51)$.

Results. We found that reading comprehension performance, WM and inhibition increased significantly with age. Further, a partial correlation showed that reading comprehension is associated with: verbal skills, WM and inhibition. Lastly, group difference testing indicates that students with high and low comprehension performance differ significantly in verbal skills and executive functioning in the 12/13 age group. In the 17/18 age group, however, they only differed significantly in terms of verbal skills.

Conclusion. Reading comprehension, WM and inhibition improve during adolescence. However, the relationship among these abilities differs by age group.

Keywords: Working memory, inhibitory processes, reading comprehension, adolescence. 


\section{Introducción}

La comprensión lectora (CL) es una variable cognitiva compleja que constituye uno de los problemas fundamentales en el estudio de la cognición humana (Kintsch, 1988). Rendimientos deficitarios en esta habilidad pueden ocasionar dificultades en distintas situaciones de la vida cotidiana, así como, repercutir en variadas situaciones de aprendizaje tanto educativas como profesionales (Cain \& Oakhill, 2007).

Se considera que el objetivo final de la comprensión es la construcción de un modelo mental interno coherente y organizado de la información contenida en el texto leído (Kintsch, 1988; Gernsbacher, 1990). Para cumplimentar con este objetivo deben llevarse a cabo un conjunto de procesamientos lingüísticos correspondientes a los niveles: léxico, morfo-sintáctico, semántico y pragmático. Sin embargo, sin la intervención de un conjunto de procesos cognitivos que permitan estos procesamientos lingüísticos no podría lograrse el objetivo de la comprensión. Por ello, ciertos investigadores plantearon que la comprensión exitosa supone el funcionamiento adecuado y la intervención de procesos cognitivos y metacognitivos de diverso orden (Cain, Oakhill \& Bryant, 2004; Gernsbacher, 1990). De este modo se considera que la CL no sólo requiere de procesos de bajo nivel de tipo bottom-up, como los procesos perceptivos que habilitan la decodificación lectora, sino que supone la intervención de procesos cognitivos de nivel superior o top-down, como la Memoria de Trabajo (MT).

Diversas investigaciones vincularon la CL y procesos cognitivos como: - el monitoreo de la lectura (Kolić-Vehovec \& Bajšanski, 2006; Oakhill, Hartt \& Samols, 2005), la MT (Abusamra, Cartoceti, Raiter \& Ferreres, 2008; Daneman \& Carpenter, 1980; De Beni, Borella \& Carretti, 2007; Swanson \& O’Conner, 2009) y la inhibición (Cain, 2006; Chiappe, Hasher \& Siegel, 2000; Gernsbacher, 1993).

De este modo, se supone que los procesamientos involucrados en la CL requieren de los procesos top-down denominados de control ejecutivo o de control cognitivo (Diamond, 2013). Estos sostienen comportamientos complejos dirigidos a metas y se consideran habilidades necesarias para regular el comportamiento, las cogniciones y las emociones de acuerdo a metas internas (Best, Miller \& Jones, 2009; Diamond, 2013; Huizinga, Dolan \& van der Molen, 2006). Actualmente, existe consenso en incluir dentro de este conjunto a la MT, la 
inhibición y la flexibilidad cognitiva (Davidson, Amso, Anderson \& Diamond, 2006, Diamond, 2013; Gilbert \& Burgess, 2008; Russell, 1999).

Asimismo, la investigación sobre los procesos de control ejecutivos ha planteado distintos patrones de desarrollo que se inician en la infancia y continúan hasta la adolescencia tardía (Blakemore \& Choudhury, 2006; Davidson et al., 2006; Gilbert \& Burgess, 2008; Hughes, 2011). Estos procesos se tornan progresivamente más eficientes e impactan de manera significativa sobre varios aspectos de la cognición y el comportamiento (Harnishfeger, 1995).

Dada la importancia que se atribuye a la MT y a la inhibición como los principales procesos ejecutivos (Russell, 1999) y a la asociación que estudios previos plantean entre cada uno de ellos y el rendimiento en CL (Borella, Carretti \& Pelegrina, 2010; Cain, 2006; CanetJuric, Urquijo, Richard’s \& Burín, 2009; Daneman \& Carpenter, 1980), esta investigación abordará ambos procesos conjuntamente. Asimismo, buscará evaluar la importancia que poseen en la explicación de las diferencias de desempeño en CL en adolescentes.

La mayoría de los trabajos empíricos presentes en la literatura se dedican a estudiar la CL y su asociación a diversos procesos ejecutivos en población infantil o adulta, dejando de lado la etapa de la adolescencia. Carretti, Borella, Cornoldi y De Beni (2009) realizan un meta-análisis en el que citan sólo dos estudios con participantes de 7 a 18 años y dos con participantes de 12 a 15 años. Sin embargo, en ninguno de ellos se consideran las medidas ejecutivas tal como se plantean en este estudio.

Por lo tanto, resultaría de interés estudiar la población adolescente en relación a estas variables debido a que la adolescencia es un momento de cambios comportamentales, cognitivos y cerebrales que afectan el funcionamiento ejecutivo (Blakemore \& Choudhury, 2006; Hughes, 2011; Huizinga et al., 2006), lo que implicaría desempeños en MT e inhibición propios de esta etapa vital.

\section{Aportes de los procesos ejecutivos al desempeño en $C L$}

\section{Memoria de trabajo}

La MT es un constructo teórico que, de acuerdo a la clásica definición de Baddeley \& Hitch (1974) y Baddeley (1986), se refiere a un sistema activo de almacenamiento temporario y procesamiento simultáneo de información. El modelo de Baddeley se considera estructural 
y postula la existencia de almacenes diferenciados según el tipo de contenido procesado: la MT verbal y la MT no verbal o viso-espacial.

Baddeley (1986) considera a la MT como un sistema complejo, constituido por subsistemas que cumplen múltiples funciones. Se le atribuyen los siguientes componentes: - el ejecutivo central, encargado de los recursos del procesamiento de dos subsistemas subsidiarios; un almacén fonológico, encargado del almacenamiento de la información verbal; y una agenda o registro viso-espacial, encargada del almacenamiento visual y espacial. Baddeley \& Wilson (2000) sumaron a este modelo un buffer episódico dedicado a integrar información de diversas fuentes, es decir, multidimensional, y a construir modelos mentales. En una reciente revisión, Baddeley sugiere que las funciones del ejecutivo central podrían fraccionarse en: la habilidad de focalizar y dividir la atención entre dos estímulos importantes; la flexibilidad para cambiar las tareas en curso y, por último, la conexión o interfaz con los almacenes de memoria a largo plazo (Baddeley, 2012).

La MT es un proceso de control que se torna necesario cuando se pretenden explicar procesos cognitivos superiores como el aprendizaje, el razonamiento o la comprensión. En particular, se ha señalado que la CL requiere una MT donde depositar los productos resultantes del procesamiento de las distintas frases (proposiciones textuales e inferidas) al mismo tiempo que se realizan los procesos exigidos por la lectura y la tarea en curso (Daneman \& Carpenter, 1980; Swanson \& O’Conner, 2009). Por su parte, Kendeou, Papadopoulos y Spanoudis (2012) señalan que la MT es central para la CL ya que comprender requiere almacenar temporalmente la información, activar la nueva información proveniente de la memoria a largo plazo y del texto que se está leyendo, como también, integrar activamente dicha información.

En las últimas décadas las investigaciones han mostrado que la habilidad para mantener información en el espacio de trabajo mental mientras se realiza simultáneamente otro procesamiento (MT), se desarrolla a través de los años. Se ha afirmado que los niños y los adultos mayores poseen niveles de span, es decir de amplitud de MT, menores a los adultos jóvenes (Borella, Carretti \& De Beni, 2008; Chiappe et al., 2000; Robert, Borella, Fagot, Lecerf, $\&$ de Ribaupierre, 2009). Tal como señalan Chiappe et al. 2000 el rendimiento en MT sufre 
un incremento en su funcionalidad a través de la edad y presenta una declinación gradual a través de la adultez hacia los 50 años, siendo clave pare el desarrollo cognitivo.

En la concepción de Chiappe et al. (2000), la MT es considerada como un conjunto de recursos atencionales limitados o un espacio finito de trabajo que debe compartirse entre el procesamiento y el almacenamiento. Por tanto, si las demandas de una tarea aumentan, en el ejecutivo central quedan menos recursos disponibles para los sistemas subsidiarios.

En esta línea, Chiappe et al. (2000) retoma investigaciones de Hasher y colegas (Hasher \& Zacks, 1988; Hasher, Lustig \& Zacks, 2007; Hasher, Tonev, Lustig \& Zacks, 2001), quienes suman un aspecto clave en el estudio de las relaciones entre MT y habilidades lectoras. Estos autores hipotetizan que las diferencias individuales asociadas a las habilidades lectoras pueden deberse a un ineficiente control inhibitorio.

\section{Procesos inhibitorios}

Tal como se señaló, la inhibición es uno de los principales procesos de regulación cognitiva ya que efectúa el control activo de los contenidos cognitivos. Esto le asigna un rol clave en el aprendizaje, el desarrollo cognitivo y el desempeño académico (Hasher et al., 2007; Hasher \& Zaks, 1988).

De modo general puede señalarse que la inhibición o el control de la interferencia refiere a la supresión de estímulos que compiten cuando se realiza una determinada tarea. Esto implicaría suprimir estímulos externos que pueden entorpecer el funcionamiento cognitivo, suprimir estímulos internos que pueden interferir con las operaciones que se llevan a cabo en la MT o suprimir respuestas prepotentes o automáticas que son irrelevantes para el logro de la tarea primaria (Diamond, 2013).

Esta caracterización del proceso inhibitorio hace referencia a un constructo que se compone de un conjunto de procesos disociables entre sí (Grandjean \& Collette, 2011; Harnishfeger, 1995). Estas diferencias constatadas experimentalmente (Friedman \& Miyake, 2004) llevaron a los investigadores a postular modelos teóricos tendientes a explicarlas.

En el marco de estos modelos se encuentran los desarrollos de Hasher y Zacks, que plantean la existencia de tres procesos inhibitorios denominados: acceso, borrado y restricción (Hasher et al. 2007; Hasher et al., 2001; Hasher \& Zacks, 1988). La función de acceso es la 
encargada de controlar el ingreso de la información irrelevante a la conciencia o foco atencional. Si falla, la información irrelevante entra al foco, dificultando el procesamiento de la información relevante. El borrado es la función que se ocupa de suprimir la información irrelevante del foco atencional, que puede haber eludido el control de acceso y sólo posteriormente, haber sido reconocida como irrelevante o también puede haber sido relevante en una situación previa pero como consecuencia del cambio en los objetivos del organismo ya no lo es. Por último, la función de restricción consiste en la supresión de respuestas preponderantes pero inapropiadas.

En este contexto, diversos estudios han planteado la relación entre la comprensión del lenguaje y los procesos inhibitorios (Borella et al., 2010; Chiappe et al., 2000). Por ejemplo, se ha señalado que los procesos inhibitorios permiten mantener la conciencia libre de información irrelevante, que puede llegar a impedir la construcción de representaciones coherentes. En concordancia con ello y dada la estrecha relación entre MT e inhibición, la literatura hipotetiza que las diferencias en el rendimiento en lectura se deberían a la habilidad de restringir la atención hacia lo que es relevante. Asimismo, diversas investigaciones han trabajado sobre las diferencias entre comprendedores de alto y bajo nivel en cuanto a su capacidad para inhibir información irrelevante y actualizar información relevante (Abusamra et al., 2008; Borella et al., 2010; Canet-Juric et al., 2009; Chiappe et al. 2000).

En esta línea se considera a la comprensión como un proceso de integración activa de información. En el cual para cumplir con el objetivo de crear una representación mental coherente del texto, el lector debe sostener cierta información en la MT mientras computa las relaciones entre palabras y oraciones, a su vez, debe poder inhibir la información irrelevante y actualizar la información relevante en su MT (Cain, 2006).

\section{Fluidez lectora y habilidades verbales}

Debido a que estudios precedentes han constatado la relación entre CL/ fluidez lectora y CL/ habilidades verbales se medirán ambas variables. Esto permitirá detectar los posibles efectos sobre el rendimiento lecto-comprensivo.

En relación a la fluidez lectora, se ha comprobado que la decodificación de las palabras es una habilidad de bajo nivel que resulta necesaria pero no suficiente para el logro de la comprensión (Defior Citoler, 2000; Oakhill, Cain \& Bryant, 2003). Decodificar las palabras 
supone que el lector traduzca el código grafémico en uno fonológico u ortográfico para luego acceder al léxico mental. Se sabe que este proceso de bajo nivel tiende a automatizarse lo que permite liberar recursos cognitivos.

Si bien es esperable que la población en estudio no presente déficits en la fluidez lectora es necesario constatarlo. Esto debido a que los lectores que no decodifiquen eficazmente utilizarán mayor cantidad de recursos atencionales en este proceso lo cual irá en detrimento de los procesos de nivel superior involucrados en la comprensión (p. e. la elaboración de inferencias) (Perfetti, 2007).

En relación a las habilidades verbales, se ha establecido que tanto los conocimientos generales sobre el mundo cómo los conocimientos sobre el vocabulario poseen efectos sobre el desempeño en CL. Stahl, Hare, Sinatra y Gregori (1991) indican que las personas que poseen mayor cantidad de conocimientos previos logran mejores desempeños en CL dado que este tipo de conocimientos influiría: -facilitando la elaboración de inferencias durante la lectura; permitiendo dirigir la atención hacia la información relevante en función del dominio del conocimiento; -brindando un posible plan para el recuerdo.

\section{Objetivos del estudio}

Por lo tanto, debido a que la habilidad de comprender textos escritos se halla asociada a este conjunto de procesos ejecutivos y estos procesos presentan un curso de desarrollo que se extiende hasta la adolescencia tardía, es posible hipotetizar que el rendimiento en la habilidad de lecto-comprensión presentará rendimientos diferenciables según la edad y mostrará diferencias de acuerdo al desarrollo de la MT y la inhibición.

Por ello se propone un conjunto de objetivos: -Primero, analizar si los rendimientos en CL, MT e inhibición presentan diferencias en adolescentes de 12/13 años y de 17/18 años de la Educación Secundaria; - Segundo, evaluar las relaciones entre los rendimientos ejecutivos de MT, inhibición y el desempeño en CL; - Tercero, analizar si los grupos de alto y bajo rendimiento en CL presentan diferencias en sus correspondientes rendimientos ejecutivos.

De este modo, las hipótesis del estudio plantean lo siguiente: - El rendimiento en CL, MT e inhibición difiere de acuerdo a la pertenencia al grupo etario, es decir, a mayor edad mejor será el rendimiento en CL, MT e inhibición; - Los rendimientos en CL se hallan aso- 
ciados a los niveles de desempeño en los procesos ejecutivos de MT e inhibición; - los grupos de desempeño de alto y bajo rendimiento en CL presentan diferencias estadísticamente significativas en sus correspondientes procesos ejecutivos de MT e inhibición. Así, los participantes que obtienen puntuaciones altas en CL serán aquellos que logran buenos desempeños en las pruebas de MT y procesos inhibitorios.

\section{Método}

\section{Participantes}

Se evaluó de manera no probabilística una muestra de 104 estudiantes de ambos sexos, divididos en cuatro grupos conformados, dos de 12/13 años $(n=53)$ y dos de 17/18 años $(n=$ 51) pertenecientes a una escuela secundaria de la ciudad de Mar del Plata. Se consideraron los siguientes criterios de inclusión: alumnos que no estén bajo tratamiento psicológico y/o psiquiátrico, que no presenten antecedentes de trastornos del aprendizaje ni del desarrollo según reportes brindados por la escuela de pertenencia.

De acuerdo al rendimiento lecto-comprensivo se seleccionaron dos grupos de cada franja etaria, distribuidos en función del siguiente criterio: 1) El grupo de comprendedores con rendimiento alto, conformado por quienes mostraron un rendimiento, al menos, de un desvío estándar por encima de la media grupal (12/13: $n=12 ; 17 / 18: n=13) ; 2)$ El grupo de comprendedores con rendimiento bajo, constituido por estudiantes cuyo desempeño se ubicó al menos un desvío estándar por debajo de la media grupal $(12 / 13: n=8 ; 17 / 18: n=9)$. A su vez, los grupos presentaron el mismo nivel en sus habilidades de fluidez lectora (12/13: $t$ $(18)=2,06 ; \mathrm{p}>.05 ; 17 / 18: t(20)=0,607 ; \mathrm{p}>.05)$.

Tabla 1. Características de la muestra total $(n=104)$ y sub-muestra según punto de corte $(n=42)$.

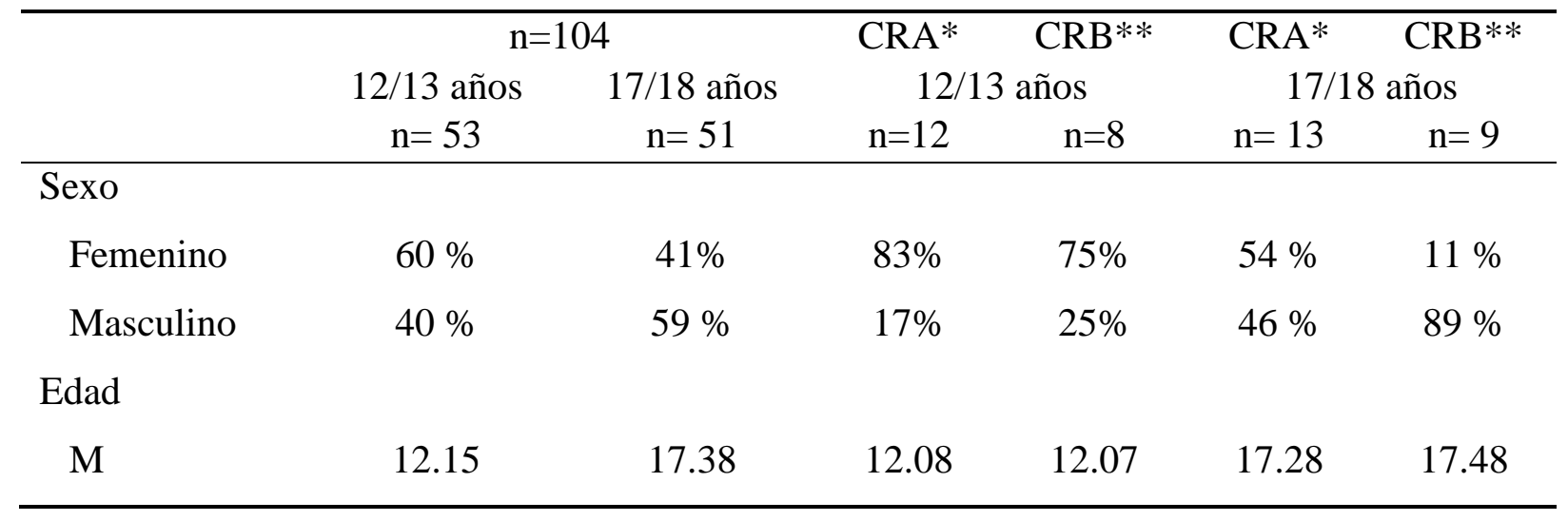




\begin{tabular}{lcccccc}
\hline DE & .277 & .448 & .017 & .035 & .417 & .499 \\
Edad mínima & 12.01 & 17.02 & 12.06 & 12.02 & 17.03 & 17.05 \\
$\begin{array}{l}\text { Edad máxi- } \\
\text { ma }\end{array}$ & 13.01 & 18.03 & 12.11 & 12.10 & 18.03 & 18.03 \\
\hline $\begin{array}{l}\text { Nota. * Comprendedores de rendimiento alto. } \\
\text { **Comprendedores de rendimiento bajo }\end{array}$ & & & & & \\
\hline
\end{tabular}

\section{Instrumentos}

Los objetivos del estudio implicaron la selección de un conjunto de tareas ad-hoc. Se administraron las siguientes pruebas:

Comprensión lectora. Consiste en la lectura silente de un texto y la resolución de diez preguntas abiertas. (Ávalos \& Díaz, 1997). La prueba se encuentra graduada según el nivel educativo, las operaciones cognitivas implicadas, la tipología textual y el grado de lecturabilidad. Por ello, se seleccionaron dos pruebas una para los alumnos de 12/13 años ("El peligro de las transfusiones"); otra para los alumnos de 17/18 años (“La salud no se mide con espejos").

Para evaluar los rendimientos ejecutivos se aplicaron las tareas de:

Memoria de Trabajo: Tarea de amplitud de palabras, perteneciente a la Batería de Evaluación de MT (AWMA) adaptada al español (Injoque-Ricle, Calero, Alloway \& Burín, 2011). La prueba aporta dos puntajes: span y puntaje total. Este último se obtiene por la sumatoria de los aciertos totales. Adicionalmente, se tomaron los errores de intrusión como medidas indirectas de inhibición. Los errores se clasifican siguiendo a Chiappe et al. (2000): palabras del ensayo en curso pero no target, que muestran una deficiencia en la inhibición como resultado de un aumento en la entrada de información irrelevante (Acceso); - palabras de listas anteriores, que reflejan interferencia proactiva que puede ser el resultado de fallos en la inhibición de borrado de información irrelevante (Borrado); - palabras extrañas que no aparecían en ningún ensayo, que reflejan deficiencias en la función de restricción de la inhibición (Restricción).

Inhibición: Test de Hayling (adaptación de Cartoceti, Sampedro, Abusamra, \& Ferreres, 2009). Evalúa los procesos de iniciación y supresión verbal. El instrumento consta de dos secciones diferentes. En la primera, el sujeto debe completar 15 oraciones en que falta la última palabra, con un estímulo que sea consistente con el contexto sintáctico-semántico (respuesta de iniciación). En la segunda, se requiere el completamiento de las oraciones con 
una palabra inconsistente con el contexto oracional (respuesta de supresión). Para la variable inhibición se considera: - tiempo de latencia en la prueba de iniciación (Iniciación); - tiempo de latencia en la prueba de supresión (Supresión); - puntaje de inhibición, este ítem asigna un puntaje que pena la proximidad semántica de la respuesta con la oración propuesta es decir, evalúa la dificultad de inhibir la respuesta dominante (Restricción).

Fluidez lectora: Sub-test de la E.N.I., Velocidad de lectura de un texto en voz alta (Matute, Rosselli, Ardila, \& Ostrosky-Solís, 2007). Se presenta al sujeto un texto (Tontolobo y el carnero) que debe leer en voz alta. Se computa el tiempo total de lectura. Para ambas franjas etarias se utilizó la misma tarea.

Habilidades Verbales: Se utilizaron los Sub-test de vocabulario del WISC III (Wechsler, 1991) y del WAIS (Wechsler, 2002), según la edad de los participantes. La tarea consiste en solicitar el significado de un conjunto de palabras. Tanto los estímulos como las respuestas son de modalidad verbal. La cantidad de palabras es 33 y la prueba se interrumpe después de 4 puntajes consecutivos de 0 puntos. El criterio de puntuación consiste en asignar 0,1 o 2 puntos según la calidad de la respuesta. Los principios generales de puntuación consideran que la respuesta es de 2 puntos si demuestra buena comprensión de la palabra (p.e. un buen sinónimo); es de 1 punto si no es incorrecta pero presenta un pobre contenido (p.e. un sinónimo vago o impreciso); le corresponde 0 punto cuando es errónea.

\section{Procedimiento}

Se solicitó el consentimiento informado a los padres o cuidadores de los participantes donde se explicitaban los objetivos y las tareas de investigación. Asimismo, en el momento de la administración los alumnos debieron asentir formar parte del estudio, pudiendo interrumpir su participación en el momento que así lo desearan. Se explicó detalladamente en qué consistía la investigación garantizándose la confidencialidad de la información obtenida y su utilización con fines exclusivamente científicos bajo la Ley Nacional 25.326 de protección de los datos personales.

El instrumento de CL se aplicó colectivamente. En cambio, las pruebas de vocabulario, fluidez lectora, MT e inhibición se tomaron en sesiones individuales. Por último, se detectó a grupos de comprendedores según su nivel de rendimiento en CL en función de los puntos de corte estadísticos (+1 DE/-1 DE). 


\section{Análisis de datos}

Se realizó un estudio de tipo descriptivo-correlacional con hipótesis de diferencia de grupos, utilizando un diseño de investigación no experimental transversal. Los datos fueron analizados utilizando la versión número 19 del SPSS software. Especificamente, se obtuvieron las medidas de tendencia central y dispersión para analizar el desempeño de los participantes en todas las variables. Adicionalmente, se aplicó una correlación parcial para obtener la relación estadística entre la CL, MT y procesos inhibitorios controlando el efecto de la edad de los participantes. Finalmente, se aplicó el Test de Student's y un indicador del tamaño del efecto para analizar las diferencias estadísticamente significativas entre los grupos de rendimiento en comprensión lectora.

\section{Resultados}

Los estadísticos de centralidad y dispersión permiten afirmar que la franja etaria de 17/18 años presentó rendimientos más eficientes en la habilidad lecto-comprensiva, la riqueza de léxico mental, la capacidad de manipulación y procesamiento de información y la habilidad de suprimir información no pertinente.

Tabla 2. Estadísticos descriptivos de las variables evaluadas para la muestra total (n=104), según grupos de edad.

\begin{tabular}{lcccccc}
\hline & \multicolumn{2}{c}{$12 / 13$ años $(\mathrm{n}=53)$} & \multicolumn{2}{c}{$17 / 18$ años $(\mathrm{n}=51)$} \\
\hline & $\mathrm{M}(\mathrm{DE})$ & Min. & Máx. & M (DE) & Min. & Máx. \\
\hline 1.Prueba de comprensión & $18.11(3.87)$ & 8 & 26 & $25.35(2.66)$ & 19 & 29 \\
2.Habilidades verbales & $39(4.32)$ & 31 & 49 & $41.63(6.52)$ & 25 & 55 \\
3. Fluidez lectora (seg.) & $35.59(5.18)$ & 26.16 & 51.94 & $27.91(3.96)$ & 20.76 & 41.63 \\
Memoria de trabajo & & & & & & \\
4.Span & $2.49(.50)$ & 2 & 3 & $3.14(.69)$ & 2 & 5 \\
5.Puntaje total & $15.52(2.07)$ & 12 & 19.5 & $19.19(3.33)$ & 14 & 30 \\
Inhibición: & & & & & & \\
Medidas indirectas & $.13(.34)$ & 0 & 1 & $.20(.40)$ & 0 & 1 \\
$\quad$ 6. Acceso & $.51(.69)$ & 0 & 2 & $.65(.74)$ & 0 & 3 \\
$\quad$ 7. Borrado & $.34(.64)$ & 0 & 3 & $.33(.71)$ & 0 & 3 \\
$\quad$ 8. Restricción & & & & & & \\
Inhibición: & $1.42(.57)$ & .68 & 2.76 & $1.08(.18)$ & .76 & 1.53 \\
Medidas directas & 9. Iniciación (seg.) & & & & &
\end{tabular}




\begin{tabular}{lcccccc}
\hline 10. Supresión (seg.) & $2.31(1.45)$ & .63 & 7.01 & $1.42(.52)$ & .82 & 3.34 \\
11. Restricción & $3.83(2.77)$ & 0 & 12 & $1.39(2.02)$ & 0 & 10 \\
\hline
\end{tabular}

A su vez, con respecto a las medidas indirectas de inhibición se observó que el comportamiento de estos indicadores fue similar en ambos grupos de edad. Esto es, la menor cantidad de errores se presentó en la función inhibitoria de acceso, seguida luego por los errores en la función de inhibición de restricción y, por último, de borrado. Asimismo, éstos últimos, es decir, aquellos errores que señalan interferencia proactiva son los que se presentan en mayor cantidad. Sin embargo, al realizar una comparación entre franjas etarias se observó que los estudiantes mayores aumentaron los errores de intrusión correspondientes a las funciones de acceso y borrado. En principio, este hallazgo se encontraría en oposición a lo esperado al iniciar el estudio, por lo que se discutirá en las conclusiones. En lo que respecta a la función de restricción los valores indican un desempeño casi equivalente.

Para cumplimentar con el primer objetivo del estudio y establecer la existencia de diferencias estadísticamente significativas entre los desempeños de cada grupo de edad, se aplicó una prueba $t$ de Student. Posteriormente, con el objeto de evaluar la magnitud de las diferencias encontradas se utilizó un indicador del tamaño del efecto: $d$ de Cohen (MoralesVallejo, 2009) calculado a través del programa Effect Syze Calculator (Watkins, 2003). Los resultados se presentan en la Tabla 3.

Tabla 3. Prueba $t$ y tamaños del efecto $\left(E t a^{2}\right)$ sobre rendimiento en CL y procesos ejecutivos según grupos de edad $(n=104)$.

\begin{tabular}{lcccc}
\hline & $t$ & $g l$. & Sig. (bilateral) & $E^{2} a^{2}$ \\
\cline { 4 - 5 } Comprensión lectora & -11.05 & 102 & $.001^{* * *}$ & -2.17 \\
Habilidades verbales & -2.42 & 102 & $.05^{*}$ & -.47 \\
Fluidez lectora & 8.45 & 102 & $.001^{* * *}$ & 1.66 \\
MT & & & & \\
$\quad$ Span & -5.45 & 102 & $.001^{* * *}$ & -1.08 \\
$\quad$ Puntaje total & -6.76 & 102 & $.001 * * *$ & -1.32 \\
Inhibición: Medidas indirectas & & & & \\
$\quad$ Acceso & .87 & 102 & $\mathrm{NS}$ & -.18 \\
\hline
\end{tabular}




\begin{tabular}{ccccc}
\hline Borrado & -.97 & 102 & NS & -.19 \\
Restricción & .04 & 102 & NS & .01 \\
Inhibición: Medidas directas & & & & \\
Iniciación verbal & 4.06 & 102 & $.001 * * *$ & .79 \\
Supresión verbal & 4.07 & 102 & $.001 * * *$ & .81 \\
Restricción & 5.10 & 102 & $.001 * * *$ & 1.00 \\
\hline
\end{tabular}

\begin{tabular}{ll}
\hline Nota. & $* p<.05 ;$ \\
& $* * p<.01 ;$ \\
& $* * * p<.001$. \\
& NS: No significativo.
\end{tabular}

Se hallaron diferencias estadísticamente significativas en relación al desempeño en CL $(p<.001)$, habilidades verbales $(p<.05)$ y fluidez lectora $(p<.001)$. Lo mismo se observó en los rendimientos ejecutivos de MT $(\operatorname{span}=p<.001$; puntaje total $=p<.001)$ e inhibición (iniciación verbal $=p<.001 ;$ supresión verbal $=p<.001 ;$ restricción $=p<.001)$. Los tamaños del efecto se hallan en consonancia a los resultados de la prueba $t$.

Para alcanzar el segundo objetivo se aplicó una correlación parcial con la intención de obtener la relación entre las variables de estudio eliminando el efecto atribuible a la edad de los participantes. Los resultados indican que la CL se halla asociada al desempeño en habilidades verbales $(p<.01)$, MT (Span $=p<.05$; Puntaje Total $=p<.01)$ y los indicadores de inhibición: Supresión $(p<.01)$ y Restricción $(p<.01)$.

Tabla 4. Correlación parcial entre pruebas administradas controlando la variable edad $(n=104)$.

\begin{tabular}{|c|c|c|c|c|c|c|c|c|c|c|}
\hline & 2 & 3 & 4 & 5 & 6 & 7 & 8 & 9 & 10 & 11 \\
\hline $\begin{array}{l}\text { 1. Comprensión } \\
\text { lectora }\end{array}$ & $\begin{array}{l}.423 \\
* *\end{array}$ & NS & $\begin{array}{c}.217 \\
*\end{array}$ & $\begin{array}{l}.279 \\
* *\end{array}$ & NS & NS & NS & NS & $\begin{array}{c}- \\
.261 \\
* *\end{array}$ & $\begin{array}{c}- \\
.272 \\
* *\end{array}$ \\
\hline $\begin{array}{l}\text { 2. Habilidades } \\
\text { verbales }\end{array}$ & - & NS & $\begin{array}{c}.222 \\
*\end{array}$ & $\begin{array}{c}.253 \\
*\end{array}$ & NS & NS & NS & NS & NS & $\begin{array}{c}- \\
.216 \\
*\end{array}$ \\
\hline $\begin{array}{l}\text { 3.Fluidez lecto- } \\
\text { ra } \\
\text { MT }\end{array}$ & & - & NS & NS & NS & NS & NS & $\begin{array}{l}.273 \\
* *\end{array}$ & NS & NS \\
\hline 4. Span & & & - & $\begin{array}{l}.885 \\
* * *\end{array}$ & NS & NS & NS & $\begin{array}{c}- \\
.195 \\
*\end{array}$ & NS & NS \\
\hline 5. Puntaje total & & & & - & NS & NS & NS & NS & NS & $\begin{array}{c}- \\
.200 \\
*\end{array}$ \\
\hline
\end{tabular}




\begin{tabular}{|c|c|c|c|c|c|c|}
\hline 6. Acceso & - & $\mathrm{NS}$ & $\mathrm{NS}$ & $\mathrm{NS}$ & $\mathrm{NS}$ & NS \\
\hline 7. Borrado & & - & $\begin{array}{c}.217 \\
*\end{array}$ & NS & NS & NS \\
\hline 8. Restricción & & & - & NS & NS & NS \\
\hline \multicolumn{7}{|l|}{ Inhibición } \\
\hline 9.Iniciación & & & & - & $\begin{array}{c}.355 \\
* *\end{array}$ & NS \\
\hline 10.Supresión & & & & & - & $\begin{array}{c}.456 \\
* *\end{array}$ \\
\hline 11.Restricción & & & & & & - \\
\hline $\begin{array}{ll}\text { Nota. } & * p<.05 ; \\
& * * p<.01 ; \\
& * * * p<.001 . \\
& \text { NS: No signifi }\end{array}$ & & & & & & \\
\hline
\end{tabular}

En relación al tercer objetivo, se analizaron las diferencias en los rendimientos ejecutivos en función del nivel de CL. La Tabla 5 presenta los resultados obtenidos.

Tabla 5. Estadísticos descriptivos de las variables evaluadas para cada nivel de CL.

\begin{tabular}{|c|c|c|c|c|}
\hline & \multicolumn{2}{|c|}{$12 / 13$ años $(n=20)$} & \multicolumn{2}{|c|}{ 17/18 años $(\mathrm{n}=22)$} \\
\hline & $\begin{array}{c}\text { CRA } \\
n=12 \\
M(D E)\end{array}$ & $\begin{array}{c}\text { CRB } \\
n=8 \\
M(D E)\end{array}$ & $\begin{array}{c}\text { CRA } \\
\mathrm{n}=13 \\
\mathrm{M}(\mathrm{DE})\end{array}$ & $\begin{array}{c}\text { CRB } \\
n=9 \\
M(D E)\end{array}$ \\
\hline $\begin{array}{l}\text { 1. Comprensión del lenguaje } \\
\text { (Rtas. Correctas/30) }\end{array}$ & $23.53(1.11)$ & $12.75(2.05)$ & $28.23(.43)$ & $20.78(1.09)$ \\
\hline 2. Habilidades verbales & $41,33(4.07)$ & $34.75(3.54)$ & $45.7(5.23)$ & $36.22(7.69)$ \\
\hline 3. Fluidez lectora & $33.48(2.75)$ & $37.74(6.38)$ & $26.44(2.68)$ & $27.05(1.56)$ \\
\hline \multicolumn{5}{|l|}{ MT } \\
\hline 4. Span & $2.67(0.49)$ & $2.13(0.35)$ & $3.46(1.05)$ & $3(.001)$ \\
\hline 5. Puntaje total & $16.41(1.60)$ & $14(2.13)$ & $20.73(4.86)$ & $18.56(1.37)$ \\
\hline \multicolumn{5}{|l|}{$\begin{array}{l}\text { Inhibición: Medidas indi- } \\
\text { rectas }\end{array}$} \\
\hline 6. Acceso & $0.08(0.28)$ & $0.13(0.35)$ & $.08(.27)$ & $.22(.44)$ \\
\hline 7. Borrado & $0.58(0.66)$ & $0.25(0.46)$ & $.62(.76)$ & $.67(1.11)$ \\
\hline 8. Restricción & $0.33(0.65)$ & $0.25(0.46)$ & $.15(.55)$ & $.67(1.11)$ \\
\hline \multicolumn{5}{|l|}{ Inhibición: Medidas directas } \\
\hline 9. Iniciación & $1.24(0.58)$ & $1.69(0.58)$ & $1.08(.165)$ & $1.06(.109)$ \\
\hline 10. Supresión & $1.93(1.33)$ & $3.19(1.83)$ & $1.28(.192)$ & $1.36(.35)$ \\
\hline 11. Restricción & $2.50(2.81)$ & $5.50(2.20)$ & $1(1.00)$ & $1.44(1.74)$ \\
\hline
\end{tabular}


Para establecer la existencia de diferencias estadísticamente significativas se aplicó una prueba $t$ de Student y se calculó el tamaño del efecto. En los grupos de 12/13 años se hallaron diferencias estadísticamente significativas en las pruebas de habilidades verbales $(p<$ $.001)$, MT $($ Span $=p<.015$, Puntaje Total $=p<.010)$ e inhibición de restricción $(p<.021)$. En cambio, los grupos de 17/18 años difirieron significativamente en relación a las habilidades verbales $(p<.001)$.

Siguiendo las orientaciones de Cohen (en Morales-Vallejo, 2009) en la franja 12/13 años los tamaños del efecto indicaron magnitudes altas para la variable: habilidades verbales, MT, inhibición de restricción y supresión verbal. En cambio, presentaron magnitudes de efecto moderadas a leves las variables: iniciación verbal e inhibición de borrado.

Con respecto a la franja 17/18 años se observó que habilidades verbales posee un tamaño de efecto alto. Asimismo, se hallaron tamaños de efecto moderados para la medida indirecta de inhibición de restricción y de MT. Finalmente, las variables inhibición de acceso, inhibición de restricción y supresión verbal presentan tamaños de efecto leves.

\section{Discusión y Conclusiones}

La CL requiere de la interacción de un conjunto de habilidades cognitivas en pos de la obtención de una representación semántica del contenido de aquello que se lee (Kendeou, van den Broek, Helder, \& Karlsson, 2014). Por ello, este trabajo buscó evaluar un grupo de habilidades relacionadas a la comprensión con la intención de comparar los rendimientos en estos procesos según la franja etaria, observar si existente asociación entre dichas habilidades y el rendimiento lecto-comprensivo y, por último, analizar si existen diferencias en los procesos ejecutivos según el nivel de desempeño en CL.

\section{Diferencias etarias en relación a los rendimientos en CL, MT e inhibición}

En relación al primer objetivo, los resultados empíricos avalarían la hipótesis sobre las diferencias asociadas a la edad en relación a la CL, la MT y la inhibición. Es decir, los adolescentes mayores alcanzan niveles de desempeño superiores a los más pequeños en relación a la eficacia del proceso lecto-comprensivo, la capacidad o amplitud de MT y el proceso de inhibición de restricción (Davidson et al., 2006; Diamond, 2013; Huizinga et al., 2006). 
La performance significativamente mejor en CL que presentan los adolescentes de 17/18 años sugiere que realizan de un modo más óptimo los procesamientos lingüísticos (léxicos, sintácticos, semánticos y pragmáticos) y las habilidades cognitivas requeridas para construir modelos mentales internos, coherentes y organizados a partir de la información textual.

A su vez, los grupos etarios difieren en sus rendimientos ejecutivos. En relación a la MT se observó que los participantes mayores presentan una mayor amplitud o nivel de span, es decir, son más hábiles para mantener y procesar la información en su mente, mientras no está disponible perceptualmente (Diamond, 2013; Lalonde, Henry, Drouin-Germain, Nolin, \& Beauchamp, 2013). Este aumento en la capacidad de la MT otorgaría a los adolescentes 17/18 años la posibilidad de contar con una mayor cantidad de recursos cognitivos para destinar a la realización de tareas cognitivas complejas (Chiappe et al., 2000; Huizinga et al., 2006).

De igual modo la performance en inhibición muestra una mayor capacidad para detener o bloquear la respuesta automática, en este caso cognitiva, que se activa ante la realización de una tarea. En otras palabras, los adolescentes mayores serían más hábiles para: - acceder a los contenidos léxicos pertinentes; -detener los contenidos no pertinentes; - cometer menor cantidad de errores cuando se debe evitar respuestas automáticas y emitir otras acordes a los objetivos de una tarea en curso. Esta habilidad permitiría que la información irrelevante no interfiera mientras se realizan tareas cognitivas complejas y otorgaría un control cognitivo más eficiente (Harnishfeger, 1995; Robert et al., 2009).

Los resultados presentados hasta aquí se hallan en concordancia con lo señalado por Blakemore y Choudhury (2006) cuando consideran que la adolescencia es un momento del desarrollo caracterizado por cambios cerebrales, comportamentales y cognitivos. Específicamente, estas investigadoras plantearon que los cambios observados en el córtex prefrontal durante la adolescencia se manifestarían en una mejora en las habilidades ejecutivas. Los hallazgos de este estudio aportarían evidencia en torno a que la performance en las funciones ejecutivas de MT e inhibición de restricción se incrementan durante la adolescencia (Blakemore \& Choudhury, 2006). En línea con estas afirmaciones, las evidencias empíricas presentadas coinciden con lo planteado por Huizinga et al. (2006) cuando sostienen que la MT y la inhibición continúan su desarrollo durante gran parte de la adolescencia, incluso este 
autor plantea que estos procesos siguen su cambio hasta la adultez. Otro de los aspectos a resaltar es la mayor variabilidad que se observa en los desempeños en MT en los estudiantes más grandes. Esto podría indicar que la variabilidad individual en relación al rendimiento en tareas ejecutivas aumentaría a lo largo del desarrollo.

Los desempeños descriptos en las medidas indirectas de inhibición admiten algunos comentarios. En primer lugar, el aumento en la cantidad de errores en la función inhibitoria de acceso y borrado en los adolescentes más grandes aparenta ser contrario a lo esperado. Sin embargo, debe considerarse que estos errores se cometen en la ejecución de una tarea de MT. Tal como plantean Kane, Conway, Hambrick \& Engle (2007), la capacidad de la MT guarda relación con el funcionamiento del ejecutivo central. Este componente o sistema de control de la MT focaliza y divide los recursos atencionales así como es capaz de cambiar el foco atencional. En esta línea, Kane et al. (2007) indican que las personas con mayor amplitud de span presentan más cantidad de errores de intrusión ya que se ven afectados por los procesos de interferencia. Esto se debería a que estas personas se esfuerzan por retener y procesar grandes cantidades de información dedicando para esas tareas más recursos atencionales. Quienes poseen altos niveles de span utilizan estrategias para manipular gran cantidad de contenidos cognitivos por lo que tienden a verse afectados por la sobrecarga de información y, por lo tanto, por mayores niveles de interferencia. En cambio, quienes tienen rendimientos más bajos en MT sólo utilizan sus recursos para sostener cantidades limitadas de información y no los dividen entre tareas, esto hace que se vean menos afectados por la interferencia.

De igual modo, parecería que los procesos de iniciación verbal (acceso al sistema cognitivo de la respuesta verbal pertinente según el contexto sintáctico-semántico) y supresión verbal (detención de la respuesta verbal automática no pertinente) se ejecutan con mayor rapidez por parte de los estudiantes más grandes. Esta diferencia en la velocidad del procesamiento podría indicar que a medida que las personas crecen el funcionamiento en estas habilidades se tornaría más óptimo. Hughes (2011) señala que habría un incremento en la velocidad de procesamiento durante la adolescencia debido a los cambios cerebrales observados durante esta etapa vital. Las modificaciones cerebrales, dado el aumento de la sustancia blanca, generarían un intercambio de información más rápido y eficiente entre el córtex frontal y otras regiones cerebrales lo que tendría consecuencias directas sobre el nivel de funcionamiento. 
En segundo lugar, el único error de intrusión que descendió en los adolescentes mayores fue el indicador de restricción, lo cual se halla en consonancia con lo encontrado en la medida directa de este mismo mecanismo. Por ello, sumaría nueva evidencia a favor de la idea de que los adolescentes más grandes serían más hábiles para detener las respuestas automáticas y dar lugar a otras adecuadas según los objetivos de las tareas en curso (Huizinga et al., 2006; Robert et al., 2009).

\section{Relaciones entre CL, MT y procesos inhibitorios}

El segundo objetivo del estudio pretendió establecer las relaciones entre el desempeño en CL en población adolescente y los rendimientos ejecutivos de MT e inhibición. Las correlaciones parciales permiten afirmar que independientemente de la edad la CL se halla asociada a las habilidades verbales, los rendimientos en MT (Christopher et al., 2012) y el proceso de inhibición de restricción (Kieffer, Vukovic, \& Berry 2013).

En principio, la relación CL/ habilidades verbales, señalaría que acceder a un vocabulario amplio contribuiría a la construcción de modelos mentales coherentes del texto. Es decir, conocer el significado de las palabras y tener un acceso eficiente a ellos implicaría: - atribuir más rápidamente el significado a las distintas palabras y proposiciones textuales; -realizar más fácilmente las inferencias requeridas durante la lectura; - liberar recursos cognitivos necesarios para la realización de otros procesos. En esta línea, se ha postulado que la amplitud de vocabulario y conocimientos previos permitiría la construcción de representaciones semánticas del contenido textual más ricas y favorecedoras de los procesos de recuerdo (Cain, Oakhill, Barnes \& Bryant, 2001; Kendeou et al., 2014; Perfetti, 2007). Por ello, los resultados coinciden con reportes previos que indican que el vocabulario es uno de los mejores predictores de la comprensión (Canet-Juric et al., 2009; Stahl et al., 1991).

En segundo lugar, la correlación CL/ MT sumaría evidencia empírica en torno a la intervención de esta función ejecutiva durante el proceso comprensivo. Esto implicaría que la MT permitiría sostener el significado de las proposiciones textuales e inferidas mientras se llevan a cabo los procesos de integración de significado necesarios para construir representaciones semánticas coherentes y globales del texto leído. Es decir, es necesario procesar y almacenar eficientemente la información en función de la construcción mental del significado de aquello que se lee (Arrington, Kulesz, Francis, Fletcher, \& Barnes, 2014; Cain et al, 2004; Carretti et al., 2009; Christopher et al., 2012; Daneman \& Merikle, 1996). 
En tercer lugar, la relación inversa entre CL/ Inhibición, señalarían que a mayor rendimiento en comprensión, menor es el tiempo requerido para suprimir información irrelevante y menor es la dificultad de restringir las respuestas prepotentes. De este modo, la evidencia indicaría que la capacidad de inhibir información irrelevante y activar sólo la relevante está implicada en el proceso comprensivo. Algunos autores sugieren que durante la comprensión se activarían más contenidos de los necesarios y que sería el proceso inhibitorio el encargado de suprimir la interferencia en función de los objetivos de la tarea. Estos hallazgos son coincidentes con la literatura (Borella et al., 2010; Canet-Juric et al., 2009; Chiappe et al., 2000; Kieffer et al., 2013).

Por lo tanto, los resultados señalan que la CL se halla asociada a los procesos ejecutivos de MT e inhibición de restricción durante la etapa de la adolescencia. Como también muestran que contar con conocimientos previos sobre el mundo y el lenguaje sigue siendo una de las habilidades que mayor influencia ejerce sobre la lecto-comprensión (Kendeou et al., 2014).

Diferencias en los rendimientos ejecutivos de MT y procesos inhibitorios en función del nivel de $C L$

Con respecto al tercer propósito del estudio, referido a establecer diferencias entre los grupos de comprendedores en relación a indicadores ejecutivos, se encontró que los grupos de 12/13 años presentaron las siguientes características: - los comprendedores con un alto nivel de rendimiento cuentan con mejores habilidades verbales, poseen una mayor amplitud de MT y logran detener más eficientemente las respuestas dominantes pero inadecuadas; - los comprendedores de bajo nivel de rendimiento, en cambio, presentan desempeños más pobres en vocabulario, menor eficiencia en los procesos de almacenamiento y mantenimiento de la información en el foco atencional y un rendimiento más bajo en el proceso inhibitorio de restricción (Borella, \& de Ribaupierre, 2014; Canet-Juric et al., 2009; Pimperton, \& Nation, 2010).

En los grupos de comprendedores de 17/18 años, se observaron las siguientes características: - los comprendedores con un alto nivel de rendimiento poseen una mayor riqueza y amplitud de su léxico mental; - en cambio, los comprendedores de bajo nivel de rendimiento, muestran desempeños más pobres en esta habilidad (Cain, \& Oakhill, 2006). Si bien los grupos no presentan diferencias estadísticamente significativas en los rendimientos ejecutivos, 
las magnitudes de los tamaños del efecto ponen de manifiesto esta influencia. Así es como podría indicarse que los comprendedores de rendimiento alto son más hábiles para manipular y sostener información mientras realizan tareas complejas (MT) (Christopher et al., 2012) y logran detener eficientemente las respuestas dominantes pero inadecuadas (inhibición de restricción) (Arrington et al., 2014; Borella, \& de Ribaupierre, 2014).

Por lo tanto, las diferencias halladas entre los grupos de comprendedores de ambas franjas etarias indicaría que quienes alcanzan procesos lectores eficientes logran además un desarrollo óptimo de los procesos ejecutivos de MT e inhibición de restricción (Peng, Sha, \& Li, 2013). Sin embargo, deben resaltarse dos aspectos. El primero, hace referencia al peso diferencial que el funcionamiento ejecutivo ejerce sobre los distintos niveles de rendimiento en CL según la franja etaria. El segundo, consiste en la importancia que las habilidades verbales poseen sobre el rendimiento en CL. Esto admitiría algunas hipótesis.

Habría que considerar que en la franja etaria de los 12/13 años el desarrollo de los procesos ejecutivos aún se encuentra en incremento y no ha alcanzado los niveles de performance de los adultos. Por ello, podría suponerse que las habilidades cognitivas complejas como la CL serían sensibles a afectarse por este proceso en desarrollo. Esto dado el menor nivel de control que los adolescentes más pequeños serían capaces de ejercer sobre la cognición compleja. Este aspecto se reflejaría en las diferencias halladas entre comprendedores de rendimiento alto y bajo en esta edad. En cambio, en la franja de 17/18 años los procesos ejecutivos estarían cercanos a los niveles adultos y el desarrollo de los mecanismos de control parecería haberse estabilizado. De este modo, controlar la cognición sería una habilidad propia de la edad. Esto determinaría que las diferencias en los procesos lectores ya no se deban predominantemente a los procesos ejecutivos (dado que se tornaron más rápidos y controlados a la vez) sino al bagaje de conocimientos sobre el mundo y el lenguaje con que cuenta el lector. De este modo los grupos de comprendedores se diferenciarían en torno a sus habilidades verbales y sus conocimientos previos más que en sus habilidades ejecutivas.

Deben tenerse en cuenta algunas de las limitaciones de nuestro estudio. En primer lugar, debe considerarse el tamaño pequeño de las muestras de comprendedores de alto y bajo rendimiento. En segundo lugar, aunque se consideraron diferentes medidas para evaluar inhibición sería apropiado utilizar medidas directas de modalidad verbal para cada uno de los 
procesos inhibitorios. En tercer lugar, sería necesario utilizar otros análisis estadísticos más complejos como el path análisis.

Para finalizar, se podrían señalar como una posible línea de investigación el desarrollo de técnicas específicas para medir cada uno de los procesos inhibitorios. De este modo se contaría con evidencia empírica a favor de la incidencia de los distintos tipos de inhibición sobre la CL. De igual modo, tendría valor ahondar sobre la relación MT/ procesos inhibitorios y Habilidades verbales/ MT. Todo ello contribuiría al conocimiento de los procesos cognitivos complejos como la CL.

\section{Referencias}

Abusamra, V., Cartoceti, R., Raiter A., \& Ferreres A. (2008). Una perspectiva cognitiva en el estudio de la comprensión de textos. Psico Porto Alegre, PUCRS, 39(3), 352-361.

Arrington, C. N., Kulesz, P. A., Francis, D. J., Fletcher, J. M., \& Barnes, M. A. (2014). The Contribution of Attentional Control and Working Memory to Reading Comprehension and Decoding. Scientific Studies of Reading, (ahead-of-print), 1-22.

Ávalos, M., \& Díaz, A. (1997). Hacia una evaluación personalizada de la comprensión lectora. Lingüística en el aula, 1(1), pp.11-43. Universidad Nacional de Córdoba. Centro de Investigaciones Lingüísticas.

Baddeley, A. (1986). Working memory. New York: Oxford University Press.

Baddeley, A. (2012). Working memory: theories, models, and controversies. Annual review of psychology, 63, 1-29. 10.1146/annurev-psych-120710-100422

Baddeley, A., \& Hitch, G. (1974). Working memory. In Bower, G.A. The Psychology of Learning and Motivation. (pp. 47-89). New York: Academic Press.

Baddeley, A., \& Wilson, B. A. (2000). Prose recall and amnesia: implications for the structure of working memory. Neuropsychologia 40, 1737-1743.

Blakemore, S., \& Choudhury, S. (2006). Development of the adolescent brain: implications for executive function and social Cognition. Journal of Child Psychology and Psychiatry 47(3), 296-312. doi:10.1111/j.1469-7610.2006.01611.x

Best, J., Miller, P., \& Jones, L. (2009). Executive functions after age 5: Changes and correlates. Developmental Review, 29 (3), 180-200. doi: 10.1016/j.dr.2009.05.002.

Borella, E., Carretti, B., \& De Beni, R. (2008). Working memory and inhibition across the adult life-span. Acta psychologica. 128, 33-44. doi:10.1016/j.actpsy.2007.09.008 
Borella, E., Carretti, B., \& Pelegrina, S. (2010). The specific role of inhibition in reading comprehension in good and poor comprehenders. Journal of Learning Disabilities 43(6), 541-552. doi: 10.1177/0022219410371676

Borella, E., Ghisletta, P., \& de Ribaupierre, A. (2011). Age differences in text processing: the role of working memory, inhibition and processing speed. Journal of Gerontology: Psychological Sciences. doi:10.1093/geronb/GBR002

Borella, E., \& de Ribaupierre, A. (2014). The role of working memory, inhibition, and processing speed in text comprehension in children. Learning and Individual Differences. Available online 19 May 2014. doi: 10.1016/j.lindif.2014.05.001

Cain, K. (2006). Individual differences in children's memory and reading comprehension: an investigation of semantic and inhibitory deficits. Memory, 14, 553-569. doi:10.1348/000709905x67610

Cain, K., \& Oakhill, J. (2006). Profiles of children with specific reading comprehension difficulties. The British Journal of Educational Psychology, 76, 683-96. doi:10.1348/000709905X67610

Cain, K., \& Oakhill, J. (2007). Children's comprehension problems in oral and written language: A Cognitive Perspective. The Guilford Press, London.

Cain, K., Oakhill, J. V, Barnes, M., \& Bryant, P. E. (2001). Comprehension skill, inferencemaking ability, and their relation to knowledge. Memory \& cognition, 29(6), 850-9.

Cain, K., Oakhill, J., \& Bryant, P. E. (2004). Children's reading comprehension ability: Concurrent prediction by working memory, verbal ability, and component skills. Journal of Educational Psychology, 96, 31-42.

Canet-Juric, L., Urquijo, S., Richard's, M.M., \& Burin, D. (2009). Predictores cognitivos de niveles de comprensión lectora mediante análisis discriminante. International Journal of Psychological Research, 2(2), 99-111.

Carretti, B., Borella, E., Cornoldi, C., \& De Beni, R. (2009). Role of working memory in explaining the performance of individuals with specific reading comprehension difficulties: A meta-analysis. Learning and Individual Differences, 19(2), 245251. doi:10.1016/j.lindif.2008.10.002

Cartoceti, R., Sampedro, B., Abusamra, V., \& Ferreres, A. (2009). Evaluación de la iniciación y la supresión de respuesta verbal en niños. Versión infantil en español del Test de Hayling. Fonoaudiológica, 55(2), 9-24.

Chiappe, P., Hasher, L., \& Siegel, L. (2000). Working memory, inhibitory control and reading disability. Memory \& Cognition 28(1), 8-17. 
Christopher, M. E., Miyake, A., Keenan, J. M., Pennington, B., DeFries, J. C., Wadsworth, S. J., Willcutt, E., \& Olson, R. K. (2012). Predicting Word Reading and Comprehension With Executive Function and Speed Measures Across Development: A Latent Variable Analysis. Journal of Experimental Psychology: General, 141(3), 470-488 doi:10.1037/a0027375

Daneman, M., \& Carpenter P. (1980). Individual differences in working memory and reading. Journal of Verbal Learning and Verbal Behavior, 19, 450-466.

Daneman, M., \& Merikle, P. (1996). Working memory and language comprehension: A metaanalysis. Psychonomic Bulletin \& Review, 3 (4), 422-433.

Davidson, M., Amso, D., Anderson, L., \& Diamond, A. (2006). Development of cognitive control and executive functions from 4 to 13 years: Evidence from manipulations of memory, inhibition, and task switching. Neuropsychologia, 44(11), 2037.

Defior Citoler, S. (2000) Las dificultades de aprendizaje. Un enfoque cognitivo. Málaga: Aljibe.

De Beni, R., Borella, E., \& Carretti, B. (2007). Reading comprehension in aging: The role of working memory and metacomprehension. Aging, Neuropsychology, and Cognition. 14(2), 189-212. doi:10.1080/13825580500229213

Diamond, A. (2013). Executive functions. Annual review of psychology, 64, 135-68.

Friedman, N., \& Miyake, A. (2004). The relations among inhibition and interference control functions: A latent-variable analysis. Journal of Experimental Psychology: General, 133, 101-135. doi:10.1037/0096-3445.137.2.201

Gernsbacher, M. A. (1990). Language comprehension as structure building. Hillsdale, NJ: Erlbaum.

Gernsbacher, M. A. (1993). Less skilled readers have less efficient suppression mechanisms. Psychological Science, 4(5), 294-298.

Gilbert, S., \& Burgess, P. (2008). Executive function. Current Biology, 18 (3), R110.

Grandjean, J., \& Collette, F. (2011). Influence of response prepotency strength, general working memory resources, and specific working memory load on the ability to inhibit predominant responses: A comparison of young and elderly participants. Brain and cognition, 77 (2), 237-247.

Harnishfeger, K. (1995). The development of cognitive inhibition: Theories, definitions, and research evidence. In F.N. Dempster, \& C. J. Brainerd (Eds.), Interference and inhibition in cognition (pp.175-204). San Diego: Academic Press. 
Hasher, L., Lustig, C., \& Zacks, R. (2007). Inhibitory mechanisms and control of attention. In A. Conway, C. Jarrold, M. Kane, A. Miyake, A., \& J. Towse (Eds.), Variation in Working Memory (pp 227-249). New York: Oxford University Press.

Hasher, L., Tonev, S., Lustig, C., \& Zacks, R. T. (2001). Inhibitory control, environmental support, and self-initiated processing in aging. In M. Naveh-Benjamin, M. Moscovitch, \& R. L. Roediger, III (Eds.), Perspectives on Human Memory and Cognitive Aging: Essays in Honour of Fergus Craik (pp. 286-297). East Sussex, UK: Psychology Press.

Hasher, L., \& Zacks, R. (1988). Working memory, comprehension, and aging: A review and a new view. In G. H. Bower (Ed.), The Psychology of Learning and Motivation, Vol. 22 (pp. 193-225). New York: Academic Press.

Hughes, C. (2011). Changes and Challenges in 20 Years of Research Into the Development of Executive Functions. Infant and Child Development, 20, 251-271. doi: 10.1002/icd.736

Huizinga, M., Dolan, C., \& van der Molen, M. (2006). Age-related change in executive function: Developmental trends and a latent variable analysis. Neuropsychologia, 44, 2017-2036. doi:10.1016/j.neuropsychologia.2006.01.010

Injoque-Ricle, I., Calero, A.D. Alloway, T.P., \& Burín, D.I. (2011). Assesing working memory in Spanish-speaking children: Automated Working Memory Assessment battery adaptation. Learning and Individual Differences, 21, 78-84.

Kane, M., Conway, A., Hambrick, D., \& Engle, R. (2007). Variation in Working Memory Capacity as Variation in Executive Attention and Control. In J. Conway, C. Jarrold, M. Kane, A. Miyake, \& J. Towse (Eds.), Variation in Working Memory (pp. 21-48). New York: Oxford University Press.

Kendeou, P., Papadopoulos, T. C., \& Spanoudis, G. (2012). Processing demands of reading comprehension tests in young readers. Learning and Instruction, 22(5), 354-367.

Kendeou, P., van den Broek, P., Helder, A., \& Karlsson, J. (2014). A Cognitive View of Reading Comprehension: Implications for Reading Difficulties. Learning Disabilities Research \& Practice, 29(1), 10-16. doi:10.1111/ldrp.12025

Kieffer, M. J., Vukovic, R. K., \& Berry, D. (2013). Roles of Attention Shifting and Reading Comprehension. Reading Research QuarterlyQuarterly, 48(4), 333-348. doi:10.1002/rrq.54

Kintsch, W. (1988). The role of knowledge in discourse processing: A constructionintegration model. Psychological Review, 95, 163-182. 
Kolić-Vehovec, S., \& Bajšanski, I. (2006). Metacognitive strategies and reading comprehension in elementary-school students. European Journal of Psychology of Education, 21(4), 439-451.

Lalonde, G., Henry, M., Drouin-Germain, A., Nolin, P., \& Beauchamp, M. H. (2013). Assessment of executive function in adolescence: a comparison of traditional and virtual reality tools. Journal of Neuroscience Methods, 219(1), 76-82. doi:10.1016/j.jneumeth.2013.07.005

Matute, E., Rosselli, M., Ardila, A., \& Ostrosky-Solís, F. (2007). Evaluación Neuropsicológica Infantil (ENI). Manual de Aplicación. México: El Manual Moderno.

Morales-Vallejo, P. (2009). El tamaño del efecto (effect size): análisis complementarios al contraste de medias. Retrieved from: http://www.upcomillas.es/personal/peter/

Oakhill, J. V., \& Cain, K. (2003). The development of comprehension skills. In T. Nunes, \& P. Bryant (Eds.), Handbook of Children's Literacy (155-180). Dordrecht: Kluwer Academic Publishers.

Oakhill, J. V, Cain, K., \& Bryant, P. E. (2003). The dissociation of word reading and text comprehension: Evidence from component skills. Language and Cognitive Processes, 18(4), 443-468. doi: 10.1080/01690960344000008

Oakhill, J. V., Hartt, J., \& Samols, D. (2005). Levels of comprehension monitoring and working memory in good and poor comprehenders. Reading and Writing, 18, 657-686.

Peng, P., Sha, T., \& Li, B. (2013). The deficit profile of working memory, inhibition, and updating in Chinese children with reading difficulties. Learning and Individual Differences, 25, 111-117.

Perfetti, C. (2007). Reading Ability: Lexical Quality to Comprehension. Scientific Studies of Reading, 11(4), 357-383.

Pimperton, H., \& Nation, K. (2010). Suppressing irrelevant information from working memory: Evidence for domain-specific deficits in poor comprehenders. Journal of Memory and Language, 62(4), 380-391. doi:10.1016/j.jml.2010.02.005

Robert, C., Borella, E., Fagot, D., Lecerf, T., \& de Ribaupierre, A. (2009). Working memory and inhibitory control across the life span: Intrusion errors in the Reading Span Test. Memory \& Cognition, 37(3), 336-45.

Russell, J. (1999). Cognitive development as an executive process - in part: A homeopathic dose of Piaget. Development Science, 2, 247-295.

Stahl, S., Hare, V., Sinatra, R., \& Gregory, J. (1991). Defining the role of Prior Knowledge and Vocabulary in Reading Comprehension. (pp. 1-25). Illinois. 
Swanson, H., \& O'Connor, R. (2009). The role of working memory and fluency practice on the reading comprehension of students who are dysfluent readers. Journal of Learning Disabilities. 42(6), pp. 548-575.

Watkins, M. W. (2003). Effect size calculator (Computer software). State College, PA EdPsych Associates.

Wechsler, D. (1991). Test de Inteligencia para Niños WISC-III. Manual. Buenos Aires: Paidós.

Wechsler, D. (2002). Escala de Inteligencia para adultos WAIS-III. Manual. Buenos Aires: Paidós. 